\title{
Satisfacción del turista con la experiencia de compra de souvenirs: el caso de un destino insular masivo de sol y playa
}

\author{
Desiderio Gutiérrez Taño* Janet Hernández Méndez** \\ Ricardo Jesús Díaz Armas**** \\ Universidad de La Laguna (España)
}

\begin{abstract}
Resumen: En este estudio se investiga la satisfacción de compra de souvenirs en destinos turísticos masivos de sol y playa, contrastando un modelo teórico en el que se plantea la influencia de la satisfacción con los atributos de compra en la satisfacción general de compra de souvenirs. Para ello se ha utilizado la modelización de ecuaciones estructurales basadas en PLS con una muestra de 134 turistas en Tenerife que han comprado algún souvenir en su estancia en la isla. Los resultados sugieren que la satisfacción con los atributos de la tienda, las características del souvenir y la presentación del mismo influyen positivamente en la satisfacción general con la compra. No obstante, otros atributos del souvenir, tales como el valor y la funcionalidad, no determinan la satisfacción de compra global. Estos resultados contribuyen a ampliar la investigación realizada sobre la satisfacción de compra con los souvenirs teniendo en cuenta un destino diferente.
\end{abstract}

Palabras Clave: Souvenir; Satisfacción; Destino turístico; Tenerife.

Tourist satisfaction with souvenirs's shopping experience: the case of a massive insular destination of sun and beach

Abstract: This study investigates the shopping satisfaction with souvenirs in a massive insular tourist destination of sun and beach, contrasting a theoretical model where the influence of satisfaction with purchasing attributes on the overall shopping satisfaction is proposed. This article applies modelling of structural equations based on PLS with a sample of 134 tourists in Tenerife who have bought souvenirs during their stay on the island. The results suggest that satisfaction with shopping attributes of the store, the characteristics of the souvenir and its presentation influence positively overall shopping satisfaction. However, other attributes such as value and functionality, don't determine overall shopping satisfaction. These findings contribute to extend the research about tourist satisfaction with souvenir in a different destination.

Keywords: Souvenir; Satisfaction; Tourist destination; Tenerife.

\section{Introducción}

Entre las principales actividades de ocio que realizan los turistas a la hora de viajar se encuentran las compras (Law \& Au, 2000; Lehto, Cai, O’Leary, \& Huan, 2004; Li \& Cai, 2008; Murphy, Moscardo, Benckendorff, \& Pearce, 2011). Jin, Moscardo y Murphy (2017) definen la compra turística como "una actividad recreativa en la que los turistas exploran, seleccionan y compran productos para llevar a casa durante su viaje". Por tanto, estas compras no sólo influyen de forma significativa sobre los comercios minoristas sino también sobre la economía de los destinos (Lin \& Lin, 2006; Oviedo-García, Vega-Vázquez, Castellanos-Verdugo, \& Reyes-Guizar, 2016).

\footnotetext{
* Universidad de La Laguna (España); E-mail: dgtano@ull.es; https://orcid.org/0000-0002-2320-946X

** Universidad de La Laguna (España); E-mail: jhernmen@ull.es

*** Universidad de La Laguna (España); E-mail: rjdiaz@ull.es; https://orcid.org/0000-0003-0585-1616
} 
Una de las características que definen a los servicios turísticos es su carácter intangible (Serra, 2011). Por este motivo los turistas compran productos que les permiten recordar las experiencias vividas en el lugar visitado (Murphy et al., 2011; Fangxuan \& Ryan, 2018) y en cierta medida, "tangibilizar lo intangible" (Serra, 2011).

Entre las diferentes compras que pueden realizar los turistas se encuentra la compra de souvenirs (Tosun, Temizkan, Timothy, \& Fyall, 2007; Lehto, Chen, \& Silkes, 2014), tales como obras de arte, antigüedades, postales, ropa, joyas, juguetes, alimentos, libros, etc. (Swanson, 2004; Swanson \& Timothy, 2012). Los souvenirs se pueden definir como productos tangibles que permiten recordar y simbolizar una determinada experiencia (Swanson \& Timothy, 2012; Ana, Elena-Nicoleta, \& Steluta, 2014). Los turistas compran souvenirs por diferentes motivos: como regalo para amigos y familiares, como recuerdo del viaje y como prueba de que han visitado un determinado destino (Wilkins, 2011).

Este estudio responde al llamamiento realizado por Suhartanto (2018) en relación a comprobar que los hallazgos encontrados en su estudio desarrollado en Bandung (Indonesia), se puedan confirmar en otros destinos con características, tipología turística, propósito de la visita y tipo de turista diferentes. Por tanto, se pretende comprobar el modelo sugerido en un destino donde el componente cultural en la motivación de la visita no es relevante. Así, el estudio se ha desarrollado en un destino turístico de sol y playa de primer nivel mundial como es Tenerife.

Tenerife es un destino insular masivo que recibe más de 5 millones de turistas al año distribuidos a lo largo del año con muy poca estacionalidad. Entre las motivaciones que atraen la visita a Tenerife como destino de sus vacaciones predomina el clima/sol en un $83 \%$ y la tranquilidad/relax (34,19\%). Las motivaciones culturales son poco importantes (ISTAC 2017).

Para conseguir los objetivos planteados, se ha desarrollado parte de la metodología del trabajo de Suhartanto (2018) en el que se establece si existe relación entre la satisfacción con los diferentes atributos de compra del souvenir y la satisfacción global con la compra. Para ello se ha realizado una encuesta entre los turistas en diferentes puntos de venta de souvenirs de la isla. Posteriormente los datos se han analizado a través de la técnica de Partial Least Squares (PLS-SEM) con el software Smart PLS v.3.2.7. Finalmente se han obtenido una serie de conclusiones que tendrían que tener en cuenta los comercios minoristas del destino objeto de estudio, Tenerife.

\section{Revisión de la literatura}

\subsection{Satisfacción del turista con la compra de souvenir}

Wong \& Wan (2013) definen la satisfacción de las compras turísticas "como una evaluación subjetiva del turista sobre su experiencia de compra con las tiendas minoristas y la mercancía comprada durante su estancia en un destino de viaje". Por tanto, un turista estará satisfecho o no con la compra de un producto tras comparar el beneficio que percibe con el mismo y las expectativas que se había formado antes de su adquisición (Heung \& Chen, 2000; Lin \& Lin, 2006).

En diferentes investigaciones sobre la satisfacción se ha comprobado la importancia de tener en cuenta el nivel de atributo y, por tanto, analizar la satisfacción general y la satisfacción con los atributos como dos constructos diferentes (Oliver 1993; Chi \& Quo 2008). En este sentido Chi \& Quo (2008) demostraron que la satisfacción con los diferentes atributos (alojamiento, actividades y eventos, accesibilidad, entorno, etc.) influye de forma positiva en la satisfacción general del turista.

En el caso específico de las compras turísticas diferentes autores han tenido en cuenta varios atributos de los productos a la hora de determinar la actitud y/o satisfacción de compra global (Heung \& Cheng 2000; Turner \& Reisenger, 2001; Lin \& Lin, 2006; Li \& Cai, 2008; Wong \& Wan, 2013; Albayrak et al., 2016). Concretamente los estudios de Li \& Cai (2008) y Suhartanto (2018) identifican cinco factores principales de los souvenirs:

- Características del souvenir (significado cultural del souvenir, calidad del souvenir)

- Funcionalidad del souvenir (utilidad, actual)

- Valor del souvenir (singularidad, unicidad, originalidad, diseño y atractivo visual del producto...)

- Atributos de la tienda (trato y servicio recibido, ambiente, localización)

- Presentación del souvenir (precio, presentación y empaquetado, elaboración y acabado del producto) 
En el presente estudio nos centraremos en los cincos factores mencionados anteriormente para observar cuáles son las dimensiones relevantes en la contribución a la satisfacción del turista en su compra.

\subsection{Modelo de satisfacción de compra}

$\mathrm{Al}$ realizar una profunda revisión de la literatura diferentes autores han estudiado la relación que existe entre la satisfacción con diferentes atributos de compra y la satisfacción global con la compra (Heung \& Cheng, 2000; Tosun et al., 2007; Murphy et al., 2011; Wong \& Wan, 2013; Ana et al., 2014; Albayrak et al., 2016; Vega-Vázquez et al., 2017; Suhartanto, 2018). No obstante, los resultados de estos estudios muestran discrepancias a la hora de determinar cuál es el atributo que influye en mayor medida en la satisfacción de la compra turística.

En este sentido Wong \& Wan (2013) comprobaron que la satisfacción con los atributos de compra se relaciona de forma positiva con la experiencia global de compra del turista. Asimismo, los resultados de su estudio revelan que la satisfacción con el producto de servicio y el entorno (localización, decoración, variedad de productos en la tienda, etc.) son los principales determinantes que favorecen la experiencia de compra. De forma adicional la satisfacción con el entorno también cobra especial protagonismo en la investigación de Vega-Vázquez et al. (2017). Estos autores demuestran que la diferenciación del servicio (servicio a domicilio, mercancía especializada, etc.) y la satisfacción del entorno influyen de forma significativa en la satisfacción de compras turísticas.

Por otra parte, estudios como los de Tosun et al. (2007) y Ana et al. (2014) comprobaron que la autenticidad de los productos es uno de los aspectos más valorados por los turistas a la hora de comprar.

Sin embargo, otros autores demostraron que la calidad del personal es el atributo que tiene mayor influencia en la experiencia de compra. Por ejemplo, Heung \& Cheng (2000) analizaron el comportamiento de compra en Hong Kong teniendo en cuenta cuatro factores específicos. En este caso comprobaron que la dimensión con mayor peso en la experiencia de compra de los turistas es la calidad del personal, seguida del valor del producto, de la fiabilidad del producto y en último lugar de la calidad de los tangibles (accesibilidad del establecimiento, variedad de productos, etc.). Asimismo, los resultados de Murphy et al. (2011) y Albayrak et al. (2016) revelaron que el personal de ventas es uno de los aspectos con más importancia e influencia en las compras turísticas.

Para la realización del presente estudio se tiene como referencia parte del modelo teórico utilizado en Suhartanto (2018) en el que se comprueba si existe relación entre la satisfacción con cinco atributos (valor del souvenir, atributos de la tienda, características del souvenir, y, por último, atributos relacionados con la presentación y funcionalidad del souvenir) y la satisfacción de compra global del souvenir. Los resultados del trabajo de Suhartanto (2018) revelan que los atributos de la tienda y las características del souvenir influyen de forma positiva en la satisfacción general de la compra del souvenir. No obstante, otros atributos, tales como el valor, la presentación y la funcionalidad del souvenir no tienen una influencia significativa sobre la satisfacción de compra.

Suhartanto (2018) sugiere que el modelo explicativo de la satisfacción con la compra del souvenir puede estar influido por las características del destino, tipología turística, propósito de la visita y tipo de turista diferentes.

En los destinos turísticos masivos donde la motivación principal del viaje tiene que ver en mayor medida con el disfrute del clima y de la playa y no tanto con los componentes culturales de atracción en su propuesta de valor, probablemente, los efectos del "valor del souvenir" y de las "características" del mismo en la satisfacción de la compra sean menos relevantes.

Como se ha señalado, este estudio pretende comprobar que el modelo contrastado por Suhartanto (2018) en Bandung, se puede confirmar en otros destinos con propósito de visita y tipo de turista diferentes.

Por tanto, se establecen las siguientes hipótesis de investigación:

H1: La satisfacción con los atributos de valor del souvenir influye positivamente en la satisfacción de la compra.

H2: La satisfacción con los atributos de la tienda influye positivamente en la satisfacción de la compra.

H3: La satisfacción con las características del souvenir influyen positivamente en la satisfacción de la compra.

H4: La satisfacción con los atributos de presentación del souvenir influyen positivamente en la satisfacción de la compra.

H5: La satisfacción con los atributos de funcionalidad del souvenir influyen positivamente en la satisfacción de la compra 
Figura 1: Modelo de satisfacción del turista con la compra del souvenir

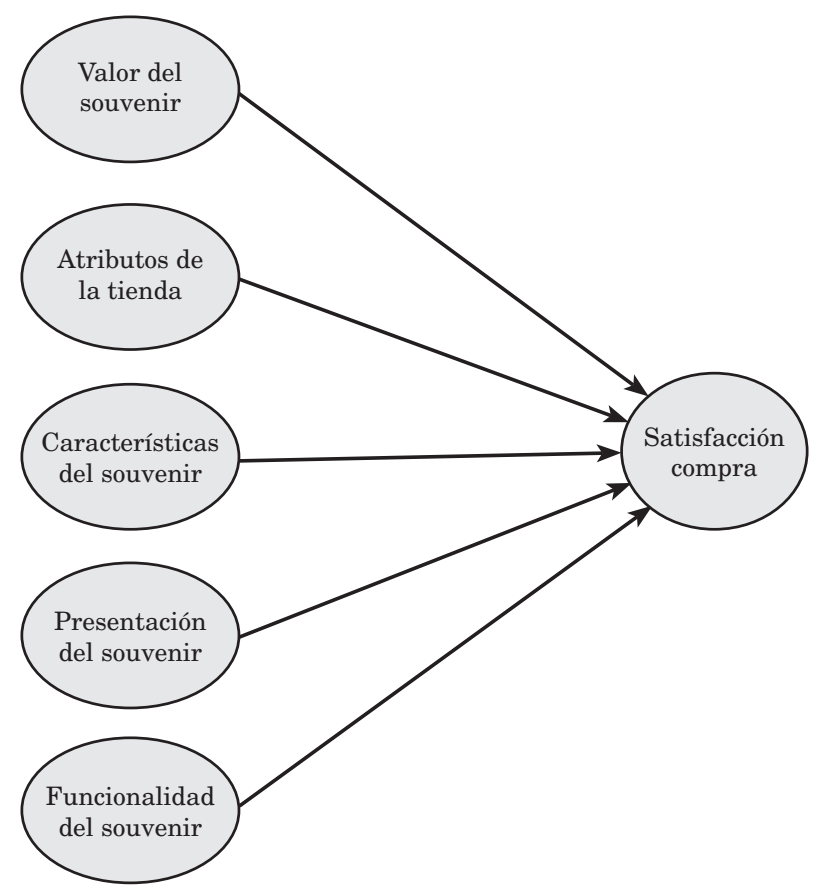

\section{Metodología de la investigación}

\subsection{Instrumento de investigación}

Para medir la satisfacción del turista con la compra del souvenir en la isla de Tenerife, se adopta la metodología del trabajo de Suhartanto (2018) llevado a cabo en Indonesia. La medición de la satisfacción del turista con la compra del souvenir se realiza a través de cinco aspectos: valor del souvenir, atributos de la tienda, aspectos del souvenir, presentación del souvenir y funcionalidad del souvenir, tomados del estudio de Li y Cai (2008). Estos consisten en 14 indicadores diferentes (ver Tabla 3) y se miden mediante una escala Likert de 5 puntos (siendo 1 muy insatisfecho y 5 muy satisfecho). Por último, se mide la satisfacción general con la compra del souvenir con una escala Likert de 5 puntos (siendo 1 muy insatisfecho y 5 muy satisfecho) y la opinión respecto a la compra en comparación con sus expectativas previas mediante una escala Likert de 5 puntos (siendo 1 mucho peor y 5 mucho mejor). Se decide utilizar la escala Likert como método de investigación por ofrecer distintas ventajas como la facilidad que presenta para su construcción, su elevada fiabilidad y el hecho de que permita establecer graduaciones o intensidades de respuesta. Entre sus inconvenientes destaca la dificultad para establecer distancias homogéneas entre los distintos ítems, ya que por ejemplo la gente suele pensar que existe una mayor diferencia entre los ítems en los extremos de la escala que entre los que se encuentran en la parte central (Kaptein et al., 2010). Asimismo, se decide usar una escala de 5 intervalos ya que numerosos estudios afirman que el número de intervalos ideal se sitúa entre 5 y 7 ítems (Alaminos \& Castejón, 2006).

Por último, se llevan a cabo distintas preguntas de clasificación para así conocer en mayor profundidad al turista. De esta forma se miden el número de veces que el turista ha visitado el destino con anterioridad, los principales motivos de elección del destino, la duración de la visita y, por último, otros aspectos más específicos como el género, la edad, la nacionalidad y la educación.

\subsection{Metodología de análisis}

Para analizar el modelo teórico propuesto y testar las hipótesis se utilizó la técnica de Partial Least Squares (PLS-SEM), con el software Smart PLS v.3.2.7 (Ringle, Wende y Becker, 2015). PLS-SEM es una técnica de modelado de ecuaciones estructurales basada en la varianza que se aplica ampliamente 
en las ciencias sociales (Henseler, Hubona y Ray, 2016). PLS-SEM es un enfoque multivariado integral para el análisis estadístico que puede examinar simultáneamente cada una de las relaciones entre las variables en un modelo conceptual, incluyendo componentes de medición y estructurales (Hair et al. 2014; Henseler, Ringle, y Sarstedt 2016; Sarstedt, Henseler, y Ringle 2011)

Por otro lado, se analizó el modelo de medición mediante la fiabilidad y la validez de los constructos reflectivos, así como el modelo estructural.

\subsection{Muestreo: perfil del entrevistado}

Para contrastar el modelo propuesto, en este estudio se realizó una encuesta entre turistas que visitaban Tenerife y que compraron algún producto de souvenir. La recogida de información se realizó mediante entrevista personal a la salida de 15 puntos de venta de souvenir distribuidos por toda la isla. El trabajo se realizó en el mes de julio de 2017, obteniendo un total de 137 entrevistas válidas, seleccionando a los individuos aleatoriamente entre los que visitaban los puntos de venta, respetando cuotas de género y edad, no de nacionalidad, pues se desconoce previamente la cuota que puede representar cada nacionalidad entre los que adquieren souvenir.

Las entrevistas se realizaron en soporte tablet y se utilizó un cuestionario en 3 idiomas (inglés, alemán y español). Para garantizar la comprensión correcta de las preguntas se realizó previamente un pretest a una muestra de 16 personas de distintas nacionalidades.

Para verificar que el tamaño de muestra es suficiente se utilizó $G^{*}$ Power (Faul et al. 2009) que sugiere que para la prueba del modelo propuesto (6 predictores) se requiere una muestra mínima de 134 individuos para una potencia estadística de 0,94 , por lo que se puede concluir con seguridad que el tamaño muestral utilizado es suficiente para los propósitos de este estudio.

Tal y como podemos observar en la Tabla 1, del total de 137 entrevistas, el 54,4\% eran mujeres y según procedencia, un 41,4\% de Reino Unido fueron, un 24,4\% de España, Alemania (12,5\%), Italia (7\%) y Canarias (5,5\%). Por otro lado, según el nivel de formación de los encuestados, destaca la educación universitaria con un 39,6\%, seguida de la Educación Primaria y Secundaria con un 29,9\%. Por último, entre las principales motivaciones para elegir Tenerife resaltan el clima, la tranquilidad y la playa, coincidiendo estos últimos datos con las estadísticas de Tenerife (Turismo de Tenerife, 2017).

Tabla 1: Características demográficas de los entrevistados

\begin{tabular}{llr}
\hline Variable & Categorias & $\%$ \\
\hline Motivo viaje & Clima o sol & $72,4 \%$ \\
& Playas & $33,6 \%$ \\
& Tranquilidad, descanso, relax & $30,6 \%$ \\
& Precio & $25,4 \%$ \\
& Paisajes & $17,2 \%$ \\
& Compras & $16,4 \%$ \\
& Seguridad & $12,7 \%$ \\
& Facilidades de traslado & $6,0 \%$ \\
& Oferta de turismo activo & $4,5 \%$ \\
& Turismo de salud & $4,5 \%$ \\
& Otros & $14,7 \%$ \\
\hline Género & Femenino & $54,5 \%$ \\
& Masculino & $45,5 \%$ \\
\hline EDAD & $18-34$ & $18,7 \%$ \\
& $35-54$ & $39,6 \%$ \\
& $55-64$ & $16,4 \%$ \\
& $>=65$ & $25,4 \%$ \\
\hline Eduación & Sin estudios & $3,7 \%$ \\
& Educación Primarios/Secundaria & $29,9 \%$ \\
& Bachillerato & $26,9 \%$ \\
& Universidad & $39,6 \%$ \\
\hline
\end{tabular}




\subsection{Tenerife en contexto}

La presente investigación se realizó en Tenerife (Islas Canarias, España). Tenerife tiene una economía de mercado avanzada basada fundamentalmente en el turismo, forma parte de la $14^{\mathrm{a}}$ economía mundial y de la $4^{\text {a }}$ economía de la Zona Euro. La isla tiene $2.034 \mathrm{Km} 2$ y una población de 904.713 habitantes (ISTAC 2018) con base poblacional cultural europea. Tiene un PIB per cápita de 20.425 , donde el sector turístico genera más del 40\% del empleo y su aportación al PIB supera el 35\% con 5,8 millones de turistas alojados en 2018 (ISTAC 2020).

\subsection{Estadísticos descriptivos}

Antes de contrastar el modelo teórico propuesto y con objeto de obtener una visión general de los resultados es conveniente realizar un análisis descriptivo (Dyer et al., 2007).

En relación con el objeto de estudio a los entrevistados se les pregunta por cuál es el souvenir adquirido (Tabla 2). En los resultados se desprende que los productos vinculados a la cultura y tradición del lugar, no son los más solicitados por los turistas que visitan Tenerife. Entre las respuestas destacan, con mayor peso, el clásico souvenir turístico, como el imán, la taza... o las prendas, complementos, souvenirs que poseen algún elemento representativo del lugar, pero que no están vinculados a los elementos de valor que generan ensoñación o recuerdo en el turista tras la visita del destino. Por el contrario, los menos solicitados son los productos de artesanía, que están más enlazados con la costumbre y realidad cultural del lugar visitado. Por tanto, los turistas visitantes de Tenerife no evidencian en su comportamiento la búsqueda de elementos que le revivan las costumbres y tradiciones del lugar visitado, o bien los productos ofertados no se ajustan a esa realidad cultural percibida del destino.

Tabla 2: Souvenir comprado

\begin{tabular}{lr}
\hline Souvenir & $\%$ \\
\hline Producto de artesania & $12,7 \%$ \\
Gastronomia (Alimentación, bebida , otros..) & $24,6 \%$ \\
Imágenes de la Isla (Fotografias, láminas...) & $23,1 \%$ \\
Ropa, complementos (Camisetas, mochilas, ...) & $29,9 \%$ \\
Souvenir-turistico" (imanes, tazas, ...) & $38,8 \%$ \\
Otro & $2,2 \%$ \\
\hline
\end{tabular}

En la Tabla 3 se pueden observar los estadísticos descriptivos (media, desviación típica y distribución porcentual de las respuestas), de cada uno de los ítems incluidos en las escalas de medida de las variables que se han utilizado para explicar la satisfacción de compra con el souvenir. En general las puntuaciones de los ítems de los diferentes apartados, tal y como se observa, muestran elevados niveles medios de satisfacción, lo que indica una distribución de las respuestas más concentrada en la parte positiva de la escala de satisfacción.

De acuerdo con los resultados obtenidos, puede apreciarse como la satisfacción con la tienda es muy alta, ya que más del $90 \%$ de las respuestas son positivas. El trato, el servicio, el ambiente o la localización, según los entrevistados, muestran resultados de elevada satisfacción. En este sentido, las tiendas visitadas cubren las expectativas que tienen los visitantes sobre los puntos de venta de souvenir.

El resto de ítems muestran valoraciones de cierta satisfacción positiva, destacando, como valoraciones menos satisfactorias, el precio y la menor superación de expectativas, pues la compra según la percepción del turista no siempre es lo que esperaba el turista.

Aunque los resultados en general son muy positivos, los entrevistados si indican algo de insatisfacción, principalmente en los ítems de singularidad, diseño, o presentación y empaquetado del souvenir. Además, un 20-25\% de las respuestas muestran que los turistas no están ni satisfechos, ni insatisfechos. La oferta y compra del souvenir no les transmite valor ni en positivo, ni en negativo. Por tanto, los establecimientos tendrían que centrar sus esfuerzos en este grupo de turistas para así tratar de mejorar la satisfacción con la compra de los souvenirs y mantener resultados de excelencia. 
Tabla 3: Estadísticas descriptivas de las variables

\begin{tabular}{|c|c|c|c|c|c|c|c|}
\hline & \multirow[b]{3}{*}{ Media } & \multirow[b]{3}{*}{$\mathrm{SD}$} & \multicolumn{5}{|c|}{ \% Respuestas } \\
\hline & & & Muy & Algo & Ni satisfecho & Algo & Muy \\
\hline & & & insatisfecho i & insatisfecho & o ni insatisfecho & satisfecho & satisfecho \\
\hline \multicolumn{8}{|l|}{ Satisfacción con el valor del souvenir } \\
\hline Singularidad, originalidad, unicidad del producto & 4,03 & 0,98 & $1 \%$ & $6 \%$ & $23 \%$ & $32 \%$ & $37 \%$ \\
\hline Diseño, atractivo visual del producto & 4,19 & 0,94 & $1 \%$ & $2 \%$ & $17 \%$ & $38 \%$ & $38 \%$ \\
\hline \multicolumn{8}{|l|}{ Satisfacción con la tienda } \\
\hline Trato y servicio recibido & 4,41 & 0,70 & $1 \%$ & $1 \%$ & $4 \%$ & $45 \%$ & $49 \%$ \\
\hline Ambiente de la tienda & 4,43 & 0,69 & $0 \%$ & $1 \%$ & $6 \%$ & $43 \%$ & $49 \%$ \\
\hline Localización de la tienda & 4,38 & 0,73 & $0 \%$ & $2 \%$ & $7 \%$ & $43 \%$ & $46 \%$ \\
\hline \multicolumn{8}{|l|}{ Satisfaccion con caracteristicas del souvenir } \\
\hline Significado cultural del souvenir & 4,01 & 1,10 & $1 \%$ & $5 \%$ & $29 \%$ & $26 \%$ & $32 \%$ \\
\hline Calidad del souvenir & 4,16 & 0,98 & $1 \%$ & $4 \%$ & $17 \%$ & $37 \%$ & $36 \%$ \\
\hline \multicolumn{8}{|l|}{ Satisfaccion con presentación del souvenir } \\
\hline Presentación y empaquetado & 4,07 & 0,92 & $0 \%$ & $4 \%$ & $25 \%$ & $37 \%$ & $31 \%$ \\
\hline Elaboración y acabado del producto & 4,13 & 1,00 & $1 \%$ & $4 \%$ & $23 \%$ & $32 \%$ & $36 \%$ \\
\hline Precio & 3,78 & 0,89 & $1 \%$ & $9 \%$ & $22 \%$ & $48 \%$ & $20 \%$ \\
\hline \multicolumn{8}{|l|}{ Satisfacción con la funcionalidad del souvenir } \\
\hline Utilidad & 4,13 & 0,89 & $0 \%$ & $3 \%$ & $22 \%$ & $36 \%$ & $37 \%$ \\
\hline Actual de moda & 4,14 & 1,22 & $0 \%$ & $4 \%$ & $36 \%$ & $21 \%$ & $20 \%$ \\
\hline \multicolumn{8}{|l|}{ Satisfacción genral con la compra } \\
\hline ¿En general, cuán de satisfecho está con la comprar? & 4,33 & 0,76 & $1 \%$ & $0 \%$ & $8 \%$ & $46 \%$ & $44 \%$ \\
\hline ¿Con respecto a lo que usted esperaba, la compra ha sido ...? & 3,64 & 0,81 & $0 \%$ & $0 \%$ & $0 \%$ & $0 \%$ & $0 \%$ \\
\hline
\end{tabular}

\subsection{Modelo de medida}

El primer paso en el proceso de análisis de un modelo de investigación que utiliza la técnica PLS consiste en evaluar el modelo de medición y tratar de verificar las características de los constructos y los elementos de medición que los representan.

Los resultados muestran que el modelo de medición cumple todos los requisitos comunes. En primer lugar, en términos de fiabilidad de los indicadores, las cargas deberían ser al menos de 0,60 e idealmente superiores a 0,7 (Chin, 1998; Henseler et al., 2009). Como se muestra en la Tabla 4, todas las cargas de los indicadores son superiores a 0,6 y en la mayoría de los casos superiores a 0,7. Además, por un lado, todas las cargas de los indicadores son significativas en el nivel de 0,001, como lo demuestran los valores t obtenidos mediante el bootstrapping, y por otro lado, todas las cargas se encuentran comprendidas en el intervalo de confianza bootstrap y también en el intervalo de confianza con sesgo corregido entre el $5 \%$ y el $95 \%$.

El criterio de la validez convergente también se cumple, ya que la varianza media extraída (AVE) de cada constructo está por encima del valor recomendado de 0,5 (Bagozzi \& Yi, 1988; Fornell \& Larcker, 1981). De forma adicional, se confirma la fiabilidad de la consistencia interna, porque la fiabilidad compuesta de todos los constructos, que muchos investigadores consideran más adecuada para PLS-SEM (por ejemplo, Garson, 2012; Hair et al., 2011; Henseler et al., 2009), excede el valor umbral recomendado de 0,70 (Bagozzi \& Yi, 1988; Nunnally, 1978). 
Tabla 4: Fiabilidad y validez de constructo

\begin{tabular}{|c|c|c|c|c|c|c|}
\hline \multirow[b]{3}{*}{ Constructo/item } & \multirow[b]{3}{*}{ Loanding } & \multirow[b]{3}{*}{ Estadisticos t } & \multicolumn{2}{|c|}{ Intervalos de confianza } & \multirow[b]{3}{*}{ CR } & \multirow[b]{3}{*}{ AVE } \\
\hline & & & & $\begin{array}{l}\text { Con sesgo } \\
\text { corregido }\end{array}$ & & \\
\hline & & & $5 \% 95 \%$ & $5 \% 95 \%$ & & \\
\hline Satisfacción con el valor del souvenir & & & & & 0,933 & 0,875 \\
\hline Singularidad, originalidad, unicidad del producto & 0,934 & $59,035 * * *$ & $0,9060,957$ & $0,9020,955$ & & \\
\hline Diseño, atractivo visual del producto & 0,936 & $51,879 * * *$ & $0,9010,960$ & $0,8950,958$ & & \\
\hline Satisfacción con la tienda & & & & & 0,845 & 0,731 \\
\hline Trato y servicio recibido & 0,939 & $47,679 * * *$ & $0,9040,965$ & $0,9010,964$ & & \\
\hline Ambiente de la tienda & 0,920 & $30,195 * * *$ & $0,8660,950$ & $0,8720,952$ & & \\
\hline Localización de la tienda & 0,650 & $5,717 * * *$ & $0,4390,798$ & $0,4160,789$ & & \\
\hline Satisfaccion con características del souvenir & & & & & 0,896 & 0,811 \\
\hline Significado cultural del souvenir & 0,890 & $35,355 * * *$ & $0,8410,923$ & $0,8360,921$ & & \\
\hline Calidad del souvenir & 0,911 & $52,133 * * *$ & $0,8790,935$ & $0,8770,934$ & & \\
\hline Satisfaccion con presentación del souvenir & & & & & 0,840 & 0,643 \\
\hline Presentación y empaquetado & 0,870 & $26,478 * * *$ & $0,8090,916$ & $0,8060,914$ & & \\
\hline Elaboración y acabado del producto & 0,899 & $46,826 * * *$ & $0,868 \quad 0,931$ & $0,8590,925$ & & \\
\hline Precio & 0,603 & $7,820 * * *$ & $0,458 \quad 0,711$ & $0,454 \quad 0,709$ & & \\
\hline Satisfacción con la funcionalidad del souvenir & & & & & 0,875 & 0,778 \\
\hline Utilidad & 0,911 & $42,854 * * *$ & $0,8710,941$ & $0,863 \quad 0,937$ & & \\
\hline Actual, de moda & 0,853 & $23,441 * * *$ & $0,783 \quad 0,900$ & $0,774 \quad 0,897$ & & \\
\hline Satisfacción genral con la compra & & & & & 0,845 & 0,731 \\
\hline ¿En general, cuán de satisfecho está con la comprar? & 0,883 & $35,598 * * *$ & $0,8410,920$ & $0,8310,916$ & & \\
\hline ¿Con respecto a lo que usted esperaba, la compra ha sido ...? & 0,825 & $25,188 * * *$ & $0,764 \quad 0,872$ & $0,7560,867$ & & \\
\hline
\end{tabular}

Significance level: ${ }^{* * *} p<0.001 ; * * p<0,01 ;{ }^{*} p<0,05$; ns no significance

La validez divergente también se verifica si se utiliza el criterio de Fornell-Larcker (Tabla 5) y las cargas cruzadas (Henseler et al., 2009). Por otro lado, la raíz cuadrada del AVE de cada constructo es mayor que sus correlaciones con cualquier otro constructo, lo que apoya la validez discriminante. Además, si se utilizan los criterios de carga cruzada, todos los indicadores tienen cargas en su respectiva construcción superiores a cualquier otra, revelando de esta manera que las construcciones son distintas (Tabla 6). En conclusión, los dos criterios apoyan la validez discriminante.

Tabla 5: Validez discriminante (Fornell-Lacker)

\begin{tabular}{lcccccr}
\hline & \multicolumn{7}{c}{ Satisfacción } \\
& Características & Presentación & Funcionalidad & compra & Tienda & Valor \\
\hline Características & $\mathbf{0 , 9 0 0}$ & & & & & \\
Presentación & 0,715 & $\mathbf{0 , 8 0 2}$ & & & & \\
Funcionalidad & 0,549 & 0,628 & $\mathbf{0 , 8 8 2}$ & & & \\
Satisfacción compra & 0,666 & 0,676 & 0,536 & $\mathbf{0 , 8 5 4}$ & & \\
Tienda & 0,296 & 0,366 & 0,267 & 0,368 & $\mathbf{0 , 8 4 7}$ & \\
Valor & 0,843 & 0,729 & 0,609 & 0,659 & 0,293 & $\mathbf{0 , 9 3 5}$ \\
\hline
\end{tabular}


Tabla 6: Cargas Cruzadas

\begin{tabular}{|c|c|c|c|c|c|c|}
\hline & \multicolumn{6}{|c|}{ Satisfacción } \\
\hline & Caracteristicas & Presentación & Funcionalidad & compra & Tienda & Valor \\
\hline Singularidad, originalidad, unicidad del producto & 0,8404 & 0,6710 & 0,5545 & 0,6113 & 0,2579 & 0,9341 \\
\hline Diseño, atractivo visual del producto & 0,7382 & 0,6930 & 0,5839 & 0,6218 & 0,2897 & 0,9364 \\
\hline Trato y servicio recibido & 0,3050 & 0,3719 & 0,2556 & 0,3948 & 0,9389 & 0,2708 \\
\hline Ambiente de la tienda & 0,2724 & 0,3470 & 0,2682 & 0,3146 & 0,9198 & 0,2716 \\
\hline Localización de la tienda & 0,1312 & 0,1517 & 0,1189 & 0,1696 & 0,6497 & 0,1959 \\
\hline Significado cultural del souvenir & 0,8897 & 0,6226 & 0,4778 & 0,5678 & 0,1615 & 0,7426 \\
\hline Calidad del souvenir & 0,9111 & 0,6631 & 0,5102 & 0,6288 & 0,3624 & 0,7756 \\
\hline Presentación y empaquetado & 0,5763 & 0,8696 & 0,5524 & 0,5487 & 0,3663 & 0,6095 \\
\hline Elaboración y acabado del producto & 0,7511 & 0,8994 & 0,5624 & 0,6687 & 0,3084 & 0,7690 \\
\hline Precio & 0,2960 & 0,6033 & 0,3754 & 0,3523 & 0,1846 & 0,2637 \\
\hline Utilidad & 0,5548 & 0,6222 & 0,9111 & 0,5235 & 0,2495 & 0,5856 \\
\hline Actual, de moda & 0,3995 & 0,4731 & 0,8528 & 0,4133 & 0,2192 & 0,4798 \\
\hline ¿En general, cuán de satisfecho está con la comprar? & 0,6150 & 0,6386 & 0,4839 & 0,8830 & 0,3256 & 0,6171 \\
\hline ¿Con respecto a lo que usted esperaba, la compra ha sido ...? & 0,5164 & 0,5066 & 0,4300 & 0,8247 & 0,3031 & 0,5015 \\
\hline
\end{tabular}

\subsection{Modelo estructural}

El segundo paso implica la evaluación del modelo estructural y de las relaciones entre los constructos según lo especificado por el modelo de investigación. La evaluación del modelo se llevó a cabo siguiendo las recomendaciones descritas por Hair et al. (2011), Hair, Sarstedt, Ringle \& Mena (2012) y Henseler, Ringle, \& Sinkovics (2009).

Uno de los pasos más importantes para evaluar el modelo interno es calcular los coeficientes de trayectoria y los niveles de significación, ya que esto permite a los investigadores confirmar o no las hipótesis propuestas. En la Figura 2 se ilustran los coeficientes del trayecto y los niveles de significación para el modelo propuesto en el presente estudio.

El valor de $\mathrm{R}^{2}$ de la variable dependiente referida a la satisfacción con la compra es del $55,2 \%$ por lo que el valor explicativo del modelo puede considerarse entre moderado y sustancial (Chin et al., 2008); débil (.19), moderado (.33) y sustancial (.67).

Por otro lado, si tenemos en cuenta las hipótesis, la H2, H3 y H4 se confirman, mientras que la H1 y la H5 no se pueden confirmar. Por tanto, se determina lo siguiente:

- La satisfacción con los atributos de la tienda influye positivamente en la satisfacción de la compra $(ß=0,121 ; \mathrm{t}=1,768)(\mathrm{H} 2)$.

- La satisfacción con las características del souvenir influye positivamente en la satisfacción de la compra $(B=0,249 ; \mathrm{t}=1,999)(\mathrm{H} 3)$.

- La satisfacción con la presentación del souvenir influye positivamente en la satisfacción de la compra $(\beta=0,285 ; \mathrm{t}=2,313)(\mathrm{H} 4)$.

Sin embargo, la hipótesis 1 en la que se establece que la satisfacción con los atributos de valor del souvenir influye positivamente en la satisfacción de la compra no se confirma. Tampoco se confirma la hipótesis 5 que sugiere que la satisfacción con los atributos de funcionalidad del souvenir influye de forma positiva en la satisfacción de la compra.

Estos resultados indican que el turista que compra los souvenirs en Tenerife, los satisfactores de la compra no son la originalidad, el diseño, la utilidad o la moda del producto. Mientras que, sí lo son el servicio y el ambiente durante su visita en la tienda, o la presentación y las características que muestra el souvenir. Estos últimos constructos explican el nivel de satisfacción del turista tras la compra del producto. Por tanto, el visitante percibe mayor satisfacción en la compra si, por un lado, el punto de venta y el servicio están orientados al beneficio buscado durante la compra y, por otro lado, el souvenir tiene una presentación ajustada a sus requerimientos. 
Figura 2: Resumen del modelo testado

Coeficientes

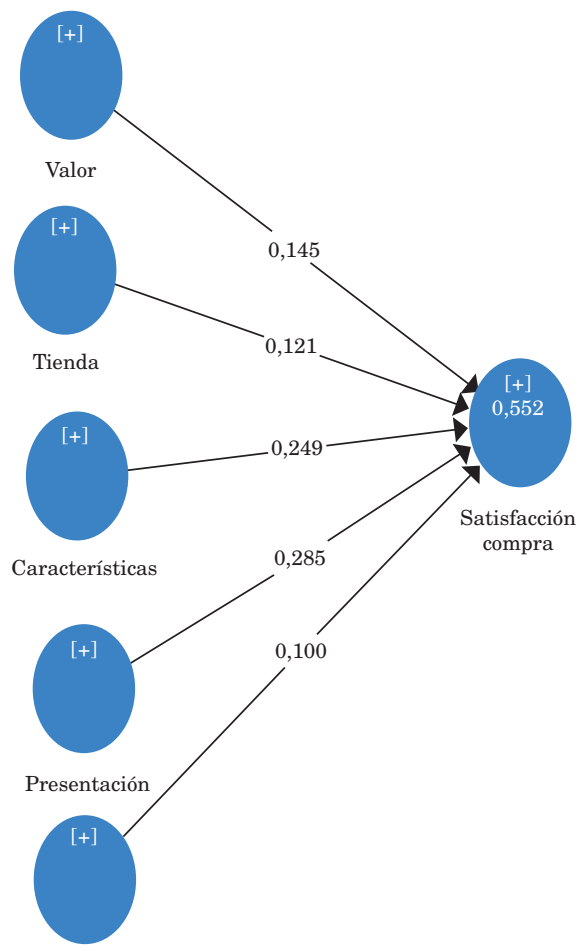

Funcionalidad
Estadísticos t

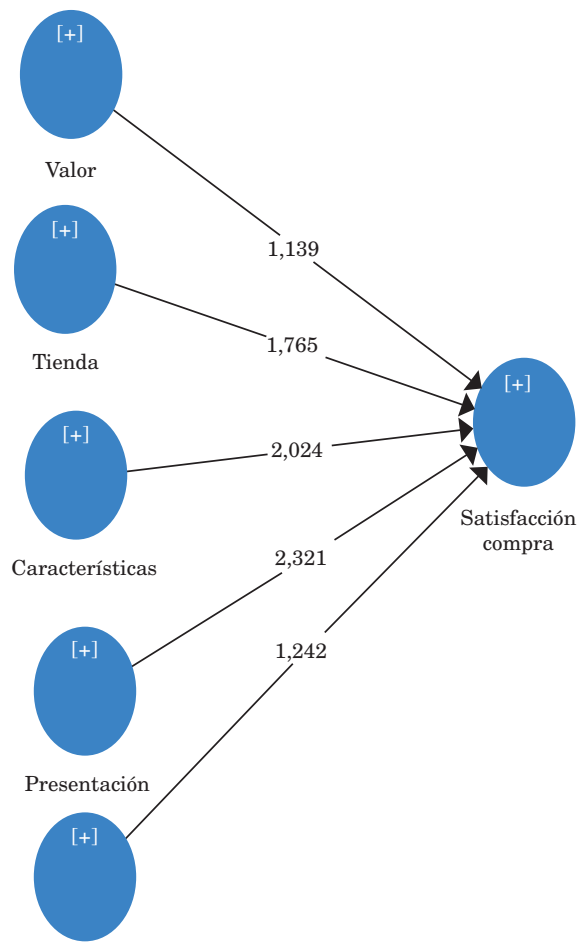

Funcionalidad

Tabla 7: Resultados del test de las hipótesis

\begin{tabular}{|c|c|c|c|c|c|c|}
\hline \multirow[b]{2}{*}{ Valor -> Satisfacción compra } & \multirow{2}{*}{$\begin{array}{r}\text { Coeficiente } \\
0,145\end{array}$} & \multirow{2}{*}{$\begin{array}{r}\text { Estadísticos t } \\
1,141 \mathrm{~ns}\end{array}$} & \multicolumn{2}{|c|}{$\begin{array}{c}\text { Intervalos de } \\
\text { confianza }\end{array}$} & \multicolumn{2}{|c|}{$\begin{array}{c}\text { Intervalos de } \\
\text { confianza con sesgc }\end{array}$} \\
\hline & & & $-0,048$ & $0,368 \mathrm{~ns}$ & $-0,081$ & $0,341 \mathrm{~ns}$ \\
\hline Tienda -> Satisfacción compra & 0,121 & $1,768 *$ & 0,001 & 0,228 & 0,004 & 0,231 \\
\hline Características -> Satisfacción compra & 0,249 & $1,999 *$ & 0,035 & 0,445 & 0,026 & 0,438 \\
\hline Presentación -> Satisfacción compra & 0,285 & $2,313 *$ & 0,077 & 0,479 & 0,089 & 0,493 \\
\hline Funcionalidad -> Satisfacción compra & 0,100 & $1,232 \mathrm{~ns}$ & $-0,033$ & $0,233 \mathrm{~ns}$ & $-0,033$ & $0,233 \mathrm{~ns}$ \\
\hline
\end{tabular}

Significance level: ${ }^{* * *} \mathrm{p}<0.001 ;{ }^{* *} \mathrm{p}<0,01 ;{ }^{*} \mathrm{p}<0,05$; ns no significance

\section{Discusión de resultados}

Diferentes autores se han centrado en estudiar las compras turísticas (Law \& Au, 2000; Lehto et al., 2004; Li \& Cai, 2008; Murphy et al., 2011; Suhartanto, 2018). La importancia de la satisfacción con las compras tiene distintos efectos, por una parte, representa un aspecto fundamental en la experiencia de los turistas en el destino (Swanson y Timothy, 2012) y, por otra parte, determina el comportamiento futuro del turista hacia el destino (Chi \& Qu, 2008; Wong \& Wan, 2013; Choi et al., 2016;). 
Aunque se ha reconocido ampliamente la importancia de las conductas de compra de los turistas en distintos destinos (Li et al., 2011; Xu \& McGehee, 2012; Ryan et al., 2013), pocos estudios han explorado las conductas de compra de recuerdos de los turistas en destinos masivos. Este estudio contribuye a la bibliografía al llenar el vacío existente en la comprensión de las conductas de compra de recuerdos de los turistas en un destino cuyo modelo turístico se caracteriza por ser masivo, de sol y playa. Por tanto, la presente investigación no incluye elementos de valor cultural y/o tradicional frente a la mayoría de estudios que se han centrado en destinos cuyos atractivos culturales son centrales (Li \& Cai, 2008; Ryan et al., 2013; Li \& Ryan, 2018).

Si comparamos los resultados de nuestro estudio con los alcanzados por Suhartanto (2018), se comprueba que los constructos que determinan la satisfacción con la compra son: los atributos de tienda y las características del souvenir. No obstante, en nuestro caso, a diferencia del estudio de Suhartanto (2018), se determina que la satisfacción con la presentación del souvenir (precio, presentación y empaquetado, elaboración y acabado del producto) también influye en la satisfacción general de la compra. Si se tiene en cuenta lo sugerido por Serra (2011), se puede decir que la adquisición de un souvenir en el destino es una forma de tangibilizar la experiencia recibida, de tener un elemento físico que permita rememorar los días de estancia o llevar el recuerdo a los familiares o amigos y, por tanto, esa representación estará vinculada a la experiencia recibida. En Tenerife la experiencia se relaciona con la tranquilidad y la desconexión en un destino de sol y playa, por lo que los elementos de presentación, empaquetado y elaboración son muy relevantes frente a otros valores que pueden ser más importantes en otro tipo de destino cultural (Li \& Cai, 2008; Ryan et al., 2013; Li \& Ryan, 2018).

De forma similar a lo obtenido por Suhartanto (2018), otros constructos, como el valor del souvenir y su funcionalidad no determinan la satisfacción con la experiencia de compra. Este hallazgo, no obstante, no está en concordancia con lo identificado por Li and Cai (2008). Esto puede deberse a las distintas motivaciones de viaje al destino como han sugerido Li y Ryan (2018) y, en nuestro caso concreto de Tenerife, los turistas le dan más importancia al clima y a las playas de la isla que a otros factores (temas culturales, compras de productos actuales...).

En consonancia con Wong \& Wan (2013) y Vega-Vázquez et al. (2017), en este modelo se determina que la gestión del espacio del punto de venta y la forma en que se entrega el souvenir son relevantes, según sus valoraciones medias y su contribución a la satisfacción. A pesar de que diferentes autores determinan que el personal es el elemento de mayor contribución (Heung \& Cheng 2000; Albayrak et al. 2016), lo cierto es que todos los ítems de este constructo (punto de venta) son relevantes.

\section{Conclusiones}

Las compras se han convertido en una de las principales actividades de los turistas cuando visitan los destinos (Li \& Cai, 2008; Murphy et al., 2011), por ello diferentes investigaciones se han centrado en analizar la satisfacción con las compras turísticas (Ana et al., 2014; Albayrak et al., 2016; Vega-Vázquez et al., 2017; Suhartanto, 2018), tratando de descubrir las variables que provocan una mayor satisfacción en la compra y, por tanto, una mayor probabilidad de que el turista evoque la visita al usar o regalar dicho souvenir.

Nuestro principal objetivo ha sido determinar cuáles son esas variables relevantes en la explicación de la satisfacción del turista con la compra del souvenir en el caso de los destinos masivos de sol y playa. Los resultados alcanzados no sólo contribuyen a ampliar las investigaciones realizadas sobre la satisfacción de compra de souvenir en diferentes destinos, sino que además ayudan a los responsables de los puntos de ventas a desarrollar e implementar diferentes acciones que permitan mejorar los beneficios de su negocio.

En esta investigación se han tenido en cuenta estudios previos (Li \& Cai, 2008; Murphy et al., 2011; Albayrak et al., 2016; Vega-Vázquez et al., 2017; Li \& Ryan, 2018) y lo propuesto por Suhartanto (2018) para desarrollar un modelo que determine y explique la satisfacción del turista con la compra de souvenirs.

La satisfacción con la compra de souvenirs se explica por la satisfacción con los atributos de la tienda, con las características del souvenir y con los atributos de presentación del souvenir. Sin embargo, no se ha podido contrastar la influencia de los atributos de valor ni de los atributos de funcionalidad del souvenir.

Los resultados de esta investigación indican que los satisfactores con la compra del souvenir están centrados en la tienda (ambiente, localización, ...) y en el servicio prestado (atención y presentación ajustada a los requerimientos del turista que lo compra), y no tanto en el producto en sí mismo (originalidad, diseño, utilidad o moda del producto). 


\section{Limitaciones}

El presente estudio cuenta con varias limitaciones por el contexto en el que se ha desarrollado. En primer lugar, sólo se ha entrevistado a los turistas que acceden a las tiendas de souvenir, sin tener en cuenta que pueden adquirir estos productos en cualquier punto de venta o localización.

Por otro lado, a pesar de que el destino considerado, Tenerife, tiene elevada importancia turística, se trata de un destino masivo de sol y playa, lo que condiciona el estudio.

En tercer lugar, aunque el presente trabajo tiene como finalidad comprobar la satisfacción del turista con la compra de souvenirs en destinos con diferentes características, tipología turística, propósito de la visita y tipo de turista, sigue siendo necesario contrastarlo en destinos de volumen, pues los resultados no son similares en todos los trabajos realizados hasta el momento.

Por último, tal y como se ha justificado en el apartado de Metodología, el tamaño muestral utilizado es suficiente para los propósitos de este estudio (Faul et al., 2009). No obstante, en futuras investigaciones se podría utilizar una muestra mayor a la hora de determinar la satisfacción del turista con la experiencia de compra de souvenirs.

\section{Bibliografía}

Alaminos, A., \& Castejón, J. L. 2006. Elaboración, análisis e interpretación de encuestas, cuestionarios y escalas de opinión. Universidad de Alicante.

Albayrak, T., Caber, M., \& Çömen, N. 2016. Tourist shopping: The relationships among shopping attributes, shopping value, and behavioral intention. Tourism Management Perspectives, 18, 98-106.

Ispas, A., Untaru, E. N., \& Ieşanu, S. 2014. Identifying a representative souvenir for a Romanian tourist destination. The case of Rupea. Annales Universitatis Apulensis: Series Oeconomica, 16(2), 193-202.

Bagozzi, R.P \& Yi, Y. 1988. On the evaluation of structural equation models. Journal of the Academy of Marketing Science, 16(1), 74-94.

Chi, C. G. Q., \& Qu, H. 2008. Examining the structural relationships of destination image, tourist satisfaction and destination loyalty: An integrated approach. Tourism management, 29(4), 624-636.

Chin, W., Peterson, R., \& Brown, S. 2008. Structural equation modeling in marketing: Some practical reminders. Journal of Marketing Theory and Practice, 16(4), 287-298.

Chin, W. W.1998. Issues and Opinion on Structure Equation Modeling. MIS Quarterly, 22(1), 7-16I.

Choi, M., Law, R., \& Heo, C. Y. 2016. Shopping destinations and trust - tourist attitudes: Scale development and validation. Tourism Management, 54, 490-501.

Dyer, P., Gursoy, D., Sharma, B., \& Carter, J. 2007. Structural modeling of resident perceptions of tourism and associated development on the Sunshine Coast, Australia. Tourism Management, 28(2),409-422

Fangxuan, L. S., \& Ryan, C. 2018. Souvenir shopping experiences: A case study of Chinese tourists in North Korea. Tourism Management, 64, 142-153.

Fornell, C., \& D. F. Larcker. 1981. Evaluating Structural Equation Models with Unobservable Variables and Measurement Error. Journal of Marketing Research, 18(1), 39-50.

Garson, G. D. 2012. Testing statistical assumptions. Asheboro, NC: Statistical Associates Publishing.

Hair, J. F., Ringle, C. M., \& Sarstedt, M. 2011. PLS-SEM: Indeed a silver bullet. Journal of Marketing theory and Practice, 19(2), 139-152

Hair, J. F., Sarstedt, M., Ringle, C. M., \& Mena, J. A. 2012. An assessment of the use of partial least squares structural equation modeling in marketing research. Journal of the Academy Marketing Science, 40, 414-433.

Hair, J. E., Hult, G. T., Ringle, C. M., \& Sarstedt, M. 2014. A primer on partial least squares structural equation modeling (PLS-SEM). Los Angeles, CA: Sage.

Henseler, J., Ringle, C. M., \& Sinkovics, R. R. 2009. The use of partial least square path modeling in international marketing. Advances in International Marketing, 20, 277-319.

Henseler, J., Ringle, C. M., \& Sarstedt, M. 2015. A new criterion for assessing discriminant validity in variance-based structural equation modeling. Journal of the Academy of Marketing Science, 40(1), $115-135$.

Henseler, J., Hubona, G., \& Ray, P. A. 2016. Using PLS path modeling in new technology research: updated guidelines. Industrial management \& data systems, 116(1), 2-20.

Heung, V. C., \& Cheng, E. 2000. Assessing tourists' satisfaction with shopping in the Hong Kong special administrative region of China. Journal of Travel Research, 38(4), 396-404. 
ISTAC. 2017. Encuesta de Gasto Turístico. Gobierno de Canarias http://www.gobiernodecanarias.org/ istac/temas_estadisticos/sectorservicios/hosteleriayturismo/demanda/C00028A.html

ISTAC. 2018. Instituto Canario de Estadística. http://www.gobiernodecanarias.org/istac/

ISTAC, 2020. Instituto Canario de Estadística. http://www.gobiernodecanarias.org/istac/

Jin, H., Moscardo, G., \& Murphy, L. 2017. Making sense of tourist shopping research: A critical review. Tourism Management, 62, 120-134.

Kaptein, M. C., Nass, C., \& Markopoulos, P. 2010, April. Powerful and consistent analysis of likert-type ratingscales. In Proceedings of the SIGCHI Conference on Human Factors in Computing Systems (pp. 2391-2394). ACM.

Kozak, M., \& Rimmington, M. 2000. Tourist satisfaction with Mallorca, Spain, as an off-season holiday destination. Journal of Travel Research, 38(1), 260-269.

Law, R., \& Au, N. 2000. Relationship modeling in tourism shopping: a decision rules induction approach". Tourism Management, 21(3), 241-249.

Lehto, X. Y., Cai, L. A., O’Leary, J. T., \& Huan, T. C. 2004. Tourist shopping preferences and expenditure behaviours: The case of the Taiwanese outbound Market”. Journal of vacation Marketing, 10(4), 320-332.

Lehto, X. Y., Chen, S. Y., \& Silkes, C. 2014. Tourist shopping style preferences. Journal of Vacation Marketing, 20(1), 3-15.

Lin, Y. H., \& Lin, K. Q. 2006. Assessing mainland Chinese visitors' satisfaction with shopping in Taiwan. Asia Pacific Journal of Tourism Research, 11(3), 247-268.

Li, M., \& Cai, L. A. 2008. "Souvenir Shopping Attitudes and Behavior Among Chinese Domestic Tourists: An Exploratory Study 中国国内游客购买旅游纪念品的态度及行为研究”. Journal of China tourism research, 4(2), 189-204.

Li, X. R., Lai, C., Harrill, R., Kline, S., \& Wang, L. 2011. When east meets west: An exploratory study on Chinese outbound tourists' travel expectations. Tourism management, 32(4), 741-749.

Li, F. S., \& Ryan, C. 2018. Souvenir shopping experiences: A case study of Chinese tourists in North Korea. Tourism Management, 64, 142-153.

Murphy, L., Moscardo, G., Benckendorff, P., \& Pearce, P. 2011. Evaluating tourist satisfaction with the retail experience in a typical tourist shopping village. Journal of retailing and Consumer Services, 18(4), 302-310.

Nunnally, J. 1978. Psychometric Theory. New York: McGraw-Hill.

Oviedo-García, M. Á., Vega-Vázquez, M., Castellanos-Verdugo, M., \& Reyes-Guizar, L. A. 2016. Tourist satisfaction and the souvenir shopping of domestic tourists: Extended weekends in Spain. Current Issues in Tourism, 19(8), 845-860.

Ringle, C. M., Wende, S. y Becker, J. M. 2015. SmartPLS 3. Boenningstedt: SmartPLS GmbH. URL: http://www.smartpls.com.

Ryan, C., Minghui, S., \& Xiaoyu, Z. 2013. Chinese Tourists and Souvenir Shopping in New Zealand. The China-New Zealand Tourism Research Centre Department of Tourism and Hospitality Management The University of Waikato Management School.

Serra, A. 2011. Marketing turístico. Madrid, España: Ediciones Pirámide.

Sarstedt, M., Henseler, J., \& Ringle, M. 2011. Multi-Group Analysis in Partial Least Squares (PLS) Path Modeling: Alternative Methods and Empirical Results. Advances in International Marketing, 22, 195-218.

Suhartanto, D. 2018. Tourist satisfaction with souvenir shopping: evidence from Indonesian domestic tourists. Current Issues in Tourism, 21(6), 663-679.

Swanson, K. K. 2004. Tourists' and retailers' perceptions of souvenirs. Journal of Vacation Marketing, 10(4), 363-377.

Swanson, K. K., \& Timothy, D. J. 2012. Souvenirs: Icons of meaning, commercialization and commoditization. Tourism Management, 33(3), 489-499.

Tosun, C., Temizkan, S. P., Timothy, D. J., \& Fyall, A. 2007. Tourist shopping experiences and satisfaction. International Journal of Tourism Research, 9(2), 87-102.

Turner, L. W., \& Reisinger, Y. 2001. Shopping satisfaction for domestic tourists. Journal of Retailing and consumer services, 8(1), 15-27.

Turismo de Tenerife. 2017. Encuesta de Turismo receptivo. Cabildo de Tenerife. https://www.webtenerife. com/investigacion/ 
Vega-Vázquez, M., Castellanos-Verdugo, M., \& Oviedo-García, M. Á. 2017. Shopping value, tourist satisfaction and positive word of mouth: the mediating role of souvenir shopping satisfaction. Current Issues in Tourism, 20(13), 1413-1430.

Wilkins, H. 2011. Souvenirs: What and why we buy. Journal of Travel Research, 50(3), 239-247.

Wong, I. A., \& Wan, Y. K. P. 2013. A systematic approach to scale development in tourist shopping satisfaction: Linking destination attributes and shopping experience. Journal of Travel Research, 52(1), 29-41.

Xu, Y., \& McGehee, N. G. 2012. Shopping behavior of Chinese tourists visiting the United States: Letting the shoppers do the talking. Tourism Management, 33(2), 427-430. 